\title{
Vaccination of pigs with the S48 strain of Toxoplasma gondii - safer meat for human consumption
}

\author{
Alison Burrells ${ }^{1}$, Julio Benavides ${ }^{1,2}$, German Cantón ${ }^{1,3}$, João L Garcia ${ }^{1,4}$, Paul M Bartley ${ }^{1}$, Mintu Nath ${ }^{5}$, \\ Jackie Thomson ${ }^{1}$, Francesca Chianini ${ }^{1}$, Elisabeth A Innes ${ }^{1}$ and Frank Katzer ${ }^{1 *}$
}

\begin{abstract}
As clinical toxoplasmosis is not considered a problem in pigs, the main reason to implement a control strategy against Toxoplasma gondii (T. gondii) in this species is to reduce the establishment of T. gondii tissue cysts in pork, consequently reducing the risk of the parasite entering the human food chain. Consumption of T. gondii tissue cysts from raw or undercooked meat is one of the main sources of human infection, with infected pork being considered a high risk. This study incorporates a mouse bioassay with molecular detection of T. gondii DNA to study the effectiveness of vaccination (incomplete S48 strain) in its ability to reduce tissue cyst burden in pigs, following oocyst (M4 strain) challenge. Results from the mouse bioassay show that 100\% of mice which had received porcine tissues from vaccinated and challenged pigs survived compared with $51.1 \%$ of mice which received tissues from non-vaccinated and challenged pigs. The presence (or absence) of T. gondii DNA from individual mouse brains also confirmed these results. This indicates a reduction in viable T. gondii tissue cysts within tissues from pigs which have been previously vaccinated with the S48 strain. In addition, the study demonstrated that the main predilection sites for the parasite were found to be brain and highly vascular muscles (such as tongue, diaphragm, heart and masseter) of pigs, while meat cuts used as human food such as chop, loin, left tricep and left semitendinosus, had a lower burden of T. gondii tissue cysts. These promising results highlight the potential of S48 strain tachyzoites for reducing the number of T. gondii tissues cysts in pork and thus improving food safety.
\end{abstract}

\section{Introduction}

The protozoan parasite Toxoplasma gondii (T. gondii) has the ability to infect all warm blooded mammals, including humans and livestock. Livestock are known intermediate hosts of $T$. gondii. Food animals such as pigs, sheep, goats and cattle become infected with the parasite, either from consumption of oocysts shed in the environment by the definitive host (felids), or in the case of pigs, from consuming other infected intermediate hosts such as rodents [1]. Following T. gondii infection, cysts form in the tissues of the animal (tissue cysts), these cysts contain the bradyzoite stage of the parasite and can survive for the lifetime of the host. The ability of tissue cysts to establish within food producing animals varies; cattle rarely have detectable tissue

\footnotetext{
* Correspondence: frank.katzer@moredun.ac.uk

${ }^{1}$ Moredun Research Institute, Pentlands Science Park, Bush Loan, Midlothian EH26 OPZ, Scotland, UK

Full list of author information is available at the end of the article
}

cysts, even in animals which have been experimentally challenged [2,3], whilst in sheep, pigs and goats, tissue cysts are more commonly identified [4]. Tissue cysts have a preference to establish in specific tissues of the host, such as liver, heart, brain, tongue, diaphragm, and skeletal muscle $[5,6]$. Viable parasites and tissue cysts have also been isolated from cuts of meat and meat products destined for human consumption from naturally and experimentally infected animals [7-11]. Consumption of raw or undercooked meat from animals containing tissue cysts is a main source of $T$. gondii infection in humans, with infected pork considered to be the major source of infection $[12,13]$.

Outdoor reared pigs are more likely to become infected with the parasite compared with those reared indoors $[14,15]$, with the main source of infection thought to be from consumption of oocyst contaminated feed, water and/or soil [16]. In addition, outdoor housing 
systems also allow pigs to come into contact with rodents and other wildlife species. Due to their omnivorous nature pigs will consume rodents or rodent cadavers as well as other small mammals and birds, which may be infected with $T$. gondii and several studies have demonstrated how rodent control programs can reduce $T$. gondii seropositivity in pigs $[1,17]$.

Although clinical toxoplasmosis in pigs is rare, and certainly not a common enough problem to warrant the commercial use of a vaccine against the parasite, the formation of tissue cysts in the muscles of infected animals can pose a significant risk for food safety and is thought to be one of the most important sources of $T$. gondii infection for humans, particularly when pork is eaten undercooked or raw $[18,19]$.

A vaccine which can reduce or eliminate the formation of infective tissues cysts in pigs would be beneficial for pork products intended for human consumption, reducing the potential public health risk from $T$. gondii infection. Previous research into the reduction of the formation of tissue cysts in pigs has included work using live and killed vaccine approaches [20-25]. It is currently unknown whether the commercially available vaccine Toxovax $^{\circ}$ (comprised of the S48 strain of T. gondii and used to protect against ovine abortion caused by the parasite), offers any protection against tissue cyst formation in livestock species. This lack of knowledge regarding the ability of S48 to protect against tissue cyst formation was highlighted, as a key knowledge gap, in a recent document produced for the Food Standards Agency [26]. The document stated that one of the gaps in the current knowledge relevant to a UK risk assessment was: "Vaccines based on live attenuated strains of tachyzoites are effective in reducing morbidity in sheep but it is not known whether vaccination has any effect on the formation of tissue cysts". Therefore, to address this knowledge gap and potentially improve food safety, this study focused on the effect of vaccination of pigs with the S48 strain of T. gondii in order to reduce tissue cyst formation. The $\$ 48$ strain was originally isolated from a case of ovine abortion in New Zealand, which after approximately 3000 passages in mice lost its ability to develop into tissue cysts (bradyzoites) in mice and oocysts in cats $[27,28]$.

The objective of this research was to evaluate the effectiveness of this live attenuated strain of $T$. gondii (S48) in its ability to reduce viable tissue cysts within porcine tissues. A reduction, or indeed elimination, of viable tissue cysts in pork would make this food source safer for human consumption. In addition, another objective was to ensure that the 548 strain used for vaccination did not persist in the pig tissues. Finally, the research aimed to provide an insight into the predilection sites for the parasite within specific porcine tissues, including tissues used within the human food chain.

\section{Materials and methods}

\section{Pig vaccination and challenge}

A total of 18 pigs, six week old Large White/Landrace cross bred pigs (Sus scrofa) of mixed gender, were divided into four groups $(G)$ depending on experimental challenge; G1 $(n=5)$ non-vaccinated and oocyst challenged animals, G2 $(n=5)$ vaccinated and oocyst challenged animals, G3 $(n=5)$ vaccinated non-challenged animals, G4 $(n=3)$ non-vaccinated and non-challenged animals. The five animals in G2 and G3 were vaccinated subcutaneously (SC) with $1.2 \times 10^{5} \quad$ S48 tachyzoites 4 weeks prior to experimental challenge (day 0) (see Table 1). Four weeks following vaccination (day 28) animals in G1 and G2 were orally challenged with $10^{3} \mathrm{~T}$. gondii oocysts of the M4 strain. During the experiment all animals were fed using a commercial pig feed and water was available ad libitum. All animal procedures complied with the Animals (Scientific Procedures) Act 1986 and were approved by the Moredun Research Institute ethics committee.

\section{Sampling and measurements}

Rectal temperatures of all pigs were monitored daily for 14 days post vaccination. At day 28 all animals were micro-chipped, iDENTICHIP ${ }^{\circ}$ with Bio-Thermo (Animalcare Ltd., York, UK), and temperature monitored for 14 days post challenge. Blood sampling was carried out weekly from days 0 to 70 of the experiment. Blood was collected from the anterior vena cava using a $2.7 \mathrm{~mL} \mathrm{~S}$ monovette serum tube with an S-monovette $20 \mathrm{G} \times 1.5$ " safety needle (Sarstedt, Leicester, UK). Blood was left to clot overnight at $4{ }^{\circ} \mathrm{C}$, tubes centrifuged for $10 \mathrm{~min}$ at $2000 \mathrm{~g}$ and serum transferred to a sterile $1.5 \mathrm{~mL}$ tube. Serum samples were stored at $-20^{\circ} \mathrm{C}$ until required. All 18 pigs were euthanised six weeks post challenge (day 70 of the experiment), by electrical stunning followed by severing of the jugular vein and exsanguination. Tissues (brain, chop, loin, left tricep, left semitedinosus, heart, masseter, tongue and diaphragm) were collected at post mortem for DNA extraction, pathology and mouse bioassay.

\begin{tabular}{|c|c|c|c|}
\hline \multirow[t]{2}{*}{ Group } & \multicolumn{2}{|c|}{ Vaccination and/or challenge } & \multirow{2}{*}{$\begin{array}{l}\text { Number of } \\
\text { animals }\end{array}$} \\
\hline & Day 0 & Day 28 & \\
\hline 1 & $\mathrm{n} / \mathrm{a}$ & $\begin{array}{l}1000 \mathrm{M} 4 \\
\text { oocysts }\end{array}$ & 5 \\
\hline 2 & $\begin{array}{l}1.2 \times 10^{5} \mathrm{~S} 48 \\
\text { tachyzoites }\end{array}$ & $\begin{array}{l}1000 \mathrm{M} 4 \\
\text { oocysts }\end{array}$ & 5 \\
\hline 3 & $\begin{array}{l}1.2 \times 10^{5} \mathrm{~S} 48 \\
\text { tachyzoites }\end{array}$ & $\mathrm{n} / \mathrm{a}$ & 5 \\
\hline 4 & $\mathrm{n} / \mathrm{a}$ & $\mathrm{n} / \mathrm{a}$ & 3 \\
\hline
\end{tabular}




\section{Mouse bioassay}

Mouse bioassay is considered the gold standard for determining the viability of $T$. gondii tissue cysts [29]. Ninety Porton mice (a minimally inbred stain), were used for the mouse bioassay. Food and water was supplied ad libitum and animals clinically monitored twice daily. Tissues from pigs in G1 (unvaccinated and oocyst challenged) and G2 (vaccinated and oocyst challenged pigs) were used for the mouse bioassay. Tissues were separated into three different $50 \mathrm{~g}$ groups, each group was based on the tissue type and divided into the following; "Brain" (50 g of brain), "Food" (a 50 g pooled sample which included $12.5 \mathrm{~g}$ each of chop, loin, left tricep and left semitendinosus), and "Other" (a $50 \mathrm{~g}$ pooled sample which included $12.5 \mathrm{~g}$ each of diaphragm, heart, tongue and masseter). These tissues were digested with acid/pepsin using a method previously described [30]. The tissue homogenate was centrifuged for $10 \mathrm{~min}$ at $1200 \mathrm{~g}$, the supernatant was poured off gently and the final pellet was resuspended in $3 \mathrm{~mL}$ sterile saline (which contained $400 \mu \mathrm{g} / \mathrm{mL}$ penicillin and 400 units/mL streptomycin). Three mice were intraperitoneally injected with $400 \mu \mathrm{L}$ of each inocula. An additional $400 \mu \mathrm{L}$ of the inocula was stored at $-20{ }^{\circ} \mathrm{C}$ for subsequent DNA extraction.

Tissues from pigs in G3 (vaccinated and non-challenged) and G4 (negative control group animals) were not included in the mouse bioassay.

Mice that showed either signs of infection, or which survived until the end of the six week bioassay, were euthanised by cervical dislocation. Blood samples were taken and brain tissue collected from each mouse. Half of each brain was stored separately in a sterile vial containing $1 \mathrm{~mL}$ PBS for DNA extraction, whist the remaining half was placed in $1 \mathrm{~mL} 10 \%$ buffered formalin for pathological examination.

\section{Detection of $T$. gondii DNA from mouse brains following bioassay}

DNA extraction followed by a $T$. gondii specific nested ITS1 PCR (n-PCR) was completed for all mice used in the bioassay $(n=90)$. DNA extraction (adapted from [31]) initially required the homogenisation of each brain sample, which was achieved by passing the material through an increasing gradient of fine gauge needles (18G, $21 \mathrm{G}$ and $25 \mathrm{G}$ needles). $900 \mu \mathrm{L}$ of Nuclei Lysis Solution (Promega, UK). Each $400 \mu \mathrm{L}$ of mouse brain homogenate was incubated overnight at $55{ }^{\circ} \mathrm{C}$, then once cooled, $300 \mu \mathrm{L}$ of Protein Precipitation Solution (Promega) was added, mixed and incubated on ice for $5 \mathrm{~min}$. The mixture was then centrifuged at $13000 \mathrm{~g}$ for $5 \mathrm{~min}$ and the resulting supernatant transferred to a $2 \mathrm{~mL}$ tube containing $900 \mu \mathrm{L}$ of isopropanol. Each tube was mixed by inversion and incubated at $-20{ }^{\circ} \mathrm{C}$ overnight. The DNA was pelleted by centrifugation at $13000 \mathrm{~g}$ for
5 min, supernatant removed and DNA pellet washed with $600 \mu \mathrm{L} \mathrm{70 \%} \mathrm{ethanol.} \mathrm{To} \mathrm{avoid} \mathrm{contamination,} \mathrm{a}$ DNA extraction control was included within each batch of extractions. The DNA pellet was centrifuged a second time and any residual ethanol removed and the pellet briefly air dried, with final re-suspension in $200 \mu \mathrm{L}$ sterile $\mathrm{H}_{2} \mathrm{O}$. To identify the presence of $T$. gondii DNA, a $T$. gondii specific ITS1 PCR was used, as previously described [32]. The ITS1 PCR was completed in triplicate for each mouse brain DNA sample. A positive control ( $T$. gondii $\mathrm{RH} \mathrm{DNA}$ ) and multiple negative controls as well as the DNA extraction control were included within each PCR run.

\section{Detection of $T$. gondii DNA from mouse bioassay inocula}

DNA was extracted from a $400 \mu \mathrm{L}$ aliquot of the acid/ pepsin porcine tissue homogenate (as previously described within this paper for DNA extraction from homogenised mouse brains). The DNA generated was tested for the presence of $T$. gondii by two different molecular methods; a qPCR targeting the T. gondii $529 \mathrm{bp}$ repeat element [10], and the T. gondii ITS1 n-PCR [32] which is described throughout this study. By using both of these methodologies, the molecular detection of the parasite can be compared between the different techniques. In addition, this also allows the results from the molecular detection of the parasite, using DNA from the inocula, to be compared to detection of the parasite within individual mouse brains from the bioassay (mouse bioassay vs. molecular detection from bioassay inocula).

\section{Molecular detection of $T$. gondii DNA from pig tissues}

DNA was extracted and tested for the presence of $T$. gondii from the following porcine tissues: brain, chop, loin, left tricep, left semitendinosus, diaphragm, heart, tongue and masseter. Aliquots of $1 \mathrm{~g}$ of each tissue for all pigs $(n=18)$ were tested individually. DNA was extracted using Precellys tubes containing ceramic beads (Peqlab, UK), followed by the T. gondii n-ITS1 specific PCR (used to detect $T$. gondii DNA), using the methodology previously described [31,32]. Each PCR was carried out in triplicate, with a positive control $(T$. gondii RH DNA), multiple negative controls and DNA extraction controls included within each PCR run.

\section{Porcine $T$. gondii IgG enzyme linked immunosorbent assay (ELISA)}

The porcine $T$. gondii IgG ELISA was adapted from a similar methodology described for detection of $T$. gondii IgG in sheep [33]. Flat-bottom 96 well polystyrene microtitration plates (MICROLON, 96K, F-form, medium binding, Greiner Bio-one, Germany) were coated with $0.1 \mathrm{~mL}$ $(2.5 \mu \mathrm{g} / \mathrm{mL})$ of $T$. gondii tachyzoite antigens prepared as previously described [34], diluted in $0.1 \mathrm{M}$ carbonate 
buffer ( $\mathrm{pH}$ 9.6) and incubated overnight at $6{ }^{\circ} \mathrm{C}$. The plates were washed 3 times with PBS-Tween $20(0.07 \mathrm{M}$ PBS/ 0.05\% Tween 20 (PBS-T)) and non-specific immune sites blocked by incubation for $1 \mathrm{~h}$ at $37^{\circ} \mathrm{C}$ with $125 \mu \mathrm{L}$ of PBS-1\% bovine serum albumin (BSA). Control and test sera were diluted 1:100 in PBS-T-1\% BSA and added to the microtitre plates in duplicate, $0.1 \mathrm{~mL}$ in each well, and incubated for $1 \mathrm{~h}$ at $37^{\circ} \mathrm{C}$. Positive and negative control sera (pool of three animals) were included in each plate. After washing, peroxidase-labeled anti-pig IgG antibody (Sigma A5670, diluted 1:10 000 in PBS-T-1\% BSA) was added $0.1 \mathrm{~mL}$ in each well and incubated for $1 \mathrm{~h}$ at $37^{\circ} \mathrm{C}$. After washing, the peroxidase activity was revealed by adding $0.1 \mathrm{~mL}$ of substrate solution (SureBlue TMB Microwell Peroxidase Substrate, KPL, Gaithersburg, MD, USA), and the reaction was stopped by adding $0.1 \mathrm{~mL}$ of $2 \mathrm{M} \mathrm{H}_{2} \mathrm{SO}_{4}$, and the optical density (OD) was read at $450 \mathrm{~nm}$ in an ELISA microplate reader (MRXII, thermo Labsystems). The average OD-value for the blank controls on a plate was subtracted from the OD-values of the sera on each plate. For control of plate-to-plate variation, the same positive and negative control sera were included on every plate and a corrected OD value was calculated for each sample as described previously [35]. A serum sample was considered to be positive when OD-value of the serum sample is greater than OD mean (from negative sera obtained from all plates, $n=64$ - negative sera from the current experiment) plus 2 standard deviation (SD from negative serum from all plates).

\section{Quantification of histopathological lesions and imunohistochemistry labelling of porcine tissues}

Methodology was carried out as described by [11]. Briefly, during histopathological examination, the numbers of glial foci and perivascular cuffs were counted for each tissue. IHC labelled slides were examined for labelled T. gondii-like structures (tachyzoites and tissue cysts). An animal was considered positive by IHC when positive labelling of tissue cysts or tachyzoites were found in at least one of its tissue sections.

\section{Murine $T$. gondii IgG ELISA}

The ID Screen Toxoplasmosis Indirect Multi-species ELISA kit (ID.vet, Montpellier, France) was used to detect IgG against $T$. gondii from mouse serum. The supplied manufacturer's instructions were followed and plates were read at $450 \mathrm{~nm}$ using the same ELISA reader as previously described. An ELISA was valid if the mean value of the positive control $\mathrm{OD}\left(\mathrm{OD}_{\mathrm{pc}}\right)$ was greater than $0.035\left(\mathrm{OD}_{\mathrm{pc}}>0.035\right)$, and if the ratio of the mean $\mathrm{OD}$ values for the positive and negative controls $\left(\mathrm{OD}_{\mathrm{pc}}\right.$ and $\left.\mathrm{OD}_{\mathrm{nc}}\right)$, were greater than $3.5\left(\mathrm{OD}_{\mathrm{pc}} / \mathrm{OD}_{\mathrm{nc}}>3.5\right)$.

The interpretation of the result was classed as percent seropositivity (SP). A sample with an SP value of $50 \%$ or higher was positive, a negative result was an SP of $40 \%$ or less, and the result classed as doubtful if the SP was between $40 \%-50 \%$.

\section{Statistical analysis}

To account for the increased variability with the mean, the weekly data on OD-value obtained from ELISA of serum samples of pigs post-vaccination were transformed using square root transformation. A repeated measures model was fitted to the transformed data incorporating treatment group, time (week post-vaccination as a categorical variable) and interaction between treatment group and time as fixed effects. The model considered a first-order autoregressive correlation structure between observations for each pig. The data on weekly rectal temperature of pigs were also analysed using a similar repeated measures model. Possible biologically interesting comparisons of differences in mean values between treatment groups were obtained using two-sided probabilities for each comparison. These probabilities were then adjusted using a False Discovery Rate (FDR) approach [36] to take into account the multiple comparisons of means so that the overall FDR was less than $5 \%$. All $p$-values in this paper refer to FDR-adjusted probabilities unless they are specified to be global $p$-values.

For the mouse bioassay, all 45 mice, that received tissues (15 mice each fed with brain, food and other tissues) from the pigs of the G2 group (vaccinated and oocyst challenged), survived until the end of the experiment (day 42), and therefore, all data in this group were censored. A total of 22 mice, inoculated with tissues from the pigs of the G1 group (unvaccinated and oocyst challenged), died at different intervals. A Kaplan-Meir survival curve was plotted to present the mean survival proportions of mice between the two groups and the equality between two survival curves was tested using G-rho family of test [37]. Similarly, the equality of survival distributions between a pair of tissues within G1 group was also tested considering pig as a stratum variable. To account for multiple comparisons, these probabilities were then adjusted using a False Discovery Rate (FDR) approach as discussed earlier.

To test the agreement of each of the two molecular tests used for detection of parasite DNA from bioassay inocula (T. gondii 529 bp qPCR and conventional PCR incorporating the $T$. gondii specific ITS1 region) with gold standard test (the detection of $T$. gondii DNA directly from mouse brains following bioassay), the data on discordant cells were tested using an exact test of a null hypothesis about the equal probability of success $(p=$ 0.5) in a Bernoulli distribution. Additionally, the data from the contingency table were used to estimate the sensitivity and specificity of both molecular tests and corresponding exact binomial confidence intervals. 
All statistical analyses were carried out using R software version 3.0.1 with appropriate $\mathrm{R}$ packages (stats, nlme, multcomp, survival, ggplot) [38].

\section{Results}

\section{Clinical observations in pigs}

Rectal temperatures of all animals were recorded from days -5 to 14 of the experiment. Estimates of mean rectal temperature and $95 \%$ confidence intervals for four treatment groups along with observed temperature on each individual pig at each day post-vaccination (until day 14) are presented in Figure 1, although this can be noted in absence of oocyst challenge (which occurred on day 28), G1 \& G4 and G2 \& G3 are identical. Due to the increasing size of the animals, the technique used for recording the temperature from days $28-42$ (daily for 14 days following oocyst challenge) was an implanted Thermochip ${ }^{\circ}$. However, this technique did provide accurate recording of the rectal temperature, probably due to the location of the chip. Temperatures fluctuated greatly resulting in vast variations of temperature readings. These readings were not reliable or informative, and therefore, were not used for further statistical analysis. On day 7 post-challenge (i.e. on day 35 of the experiment), all five animals in G2 appeared subdued/depressed for approximately $24 \mathrm{~h}$, although they remained interested in food and movement was unaffected. All other animals remained clinically normal throughout the experiment.

\section{Molecular detection of $T$. gondii from individual pig tissues}

DNA extracted from individual tissues (brain, chop, loin, left tricep, left semitendinosus, diaphragm, heart, tongue and masseter) from all pigs in G1, G2, G3 and G4 was tested for the presence of $T$. gondii DNA using the ITS1 n-PCR. Parasite DNA was not detected from any of these tissues, despite each DNA sample being tested in triplicate.

\section{Porcine $T$. gondii IgG ELISA}

Estimates of the mean transformed OD values (square root transformation) and corresponding 95\% confidence

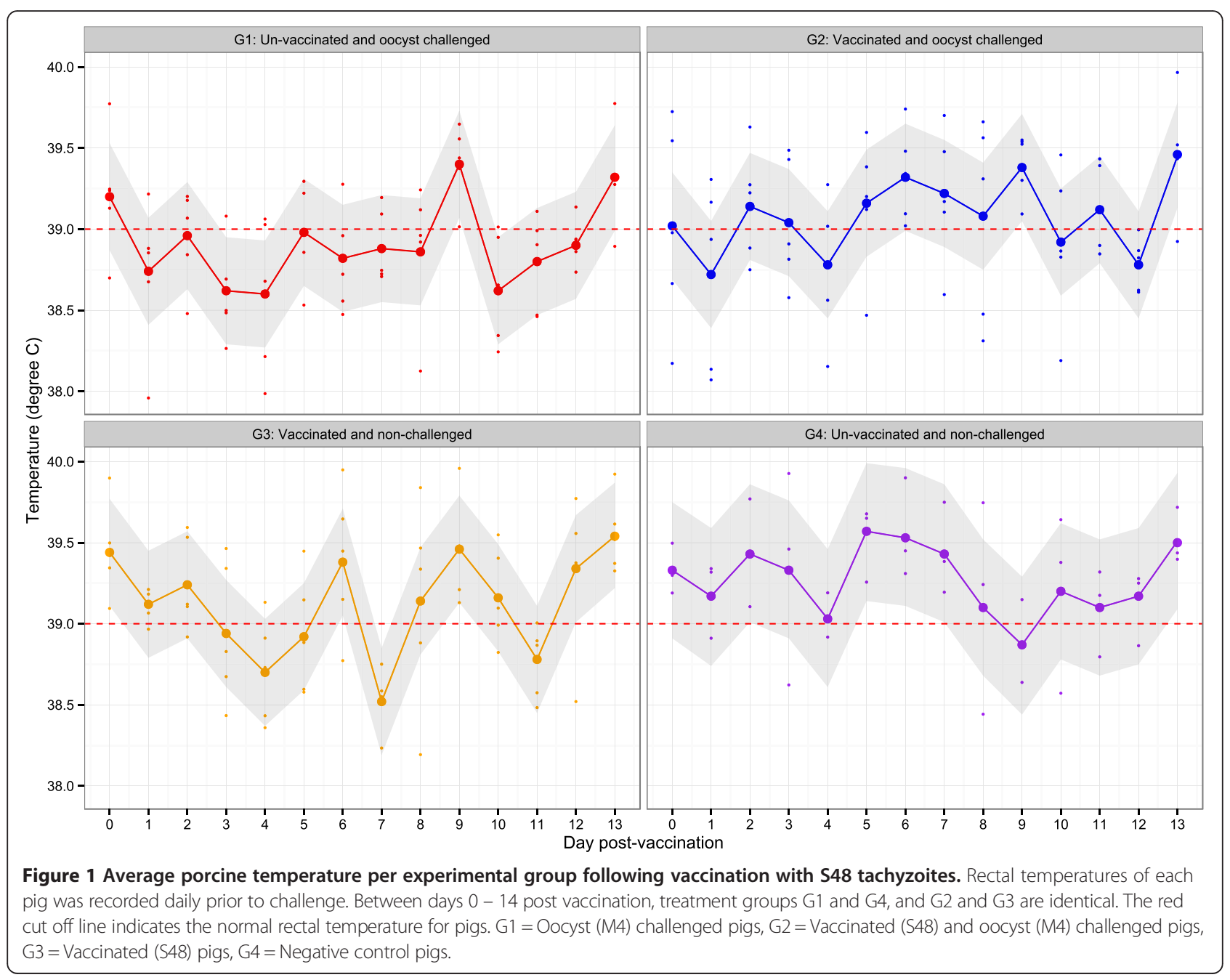


intervals for four treatment groups along with observed OD values on each individual pig at each week postvaccination are presented in Figure 2. Briefly, all pigs tested negative by ELISA for $T$. gondii IgG at the beginning of the experiment (day 0 post-vaccination), however, by day 42 post-vaccination (or 2 weeks after challenge with $T$. gondii oocysts for G1 and G2), most of the pigs (apart from the pigs in G4 - negative control animals) were $T$. gondii IgG positive (OD-value greater or equal to 0.34 , or equivalently, square root of OD-value greater or equal to 0.58; see Figure 2). The interaction effect of treatment group and time had a statistically significant (global $p<0.001)$ effect on the mean OD. The results showed that the mean OD value of pigs from G2 (pigs which were vaccinated with S48 and then challenged four weeks later with $10^{3}$ M4 T. gondii oocysts) were statistically significantly higher compared with G3 animals (pigs which were vaccinated and not challenged) at day $49(p=0.019), 63$ $(p<0.001)$ and $70(p=0.017)$ post-vaccination (Figure 2$)$. Mean OD values were higher in magnitude for the G2 group compared with G3 on day 42 and 70, but the mean differences were not statistically significant $(p>0.05)$. The negative control animals (G4) remained seronegative throughout the experiment as OD values were below the threshold during the entire experimental period (see Figure 2).

\section{Quantification of histopathological lesions and} immunohistochemistry labelling of porcine tissues

Histological examination of the brain from the infected pigs showed mild non purulent perivascular infiltration, randomly distributed, in four animals from G1 and one animal from G2, mainly formed by CD3 positive lymphocytes. Besides these lesions, mild, focal, non-specific infiltration of few mononuclear inflammatory cells, mainly lymphocytes in the lungs and the different muscles studied were found in the all the animals studied. No differences were found between the studied groups regarding these non specific changes. Immunohistochemical labelling showed few intracellular $T$. gondii

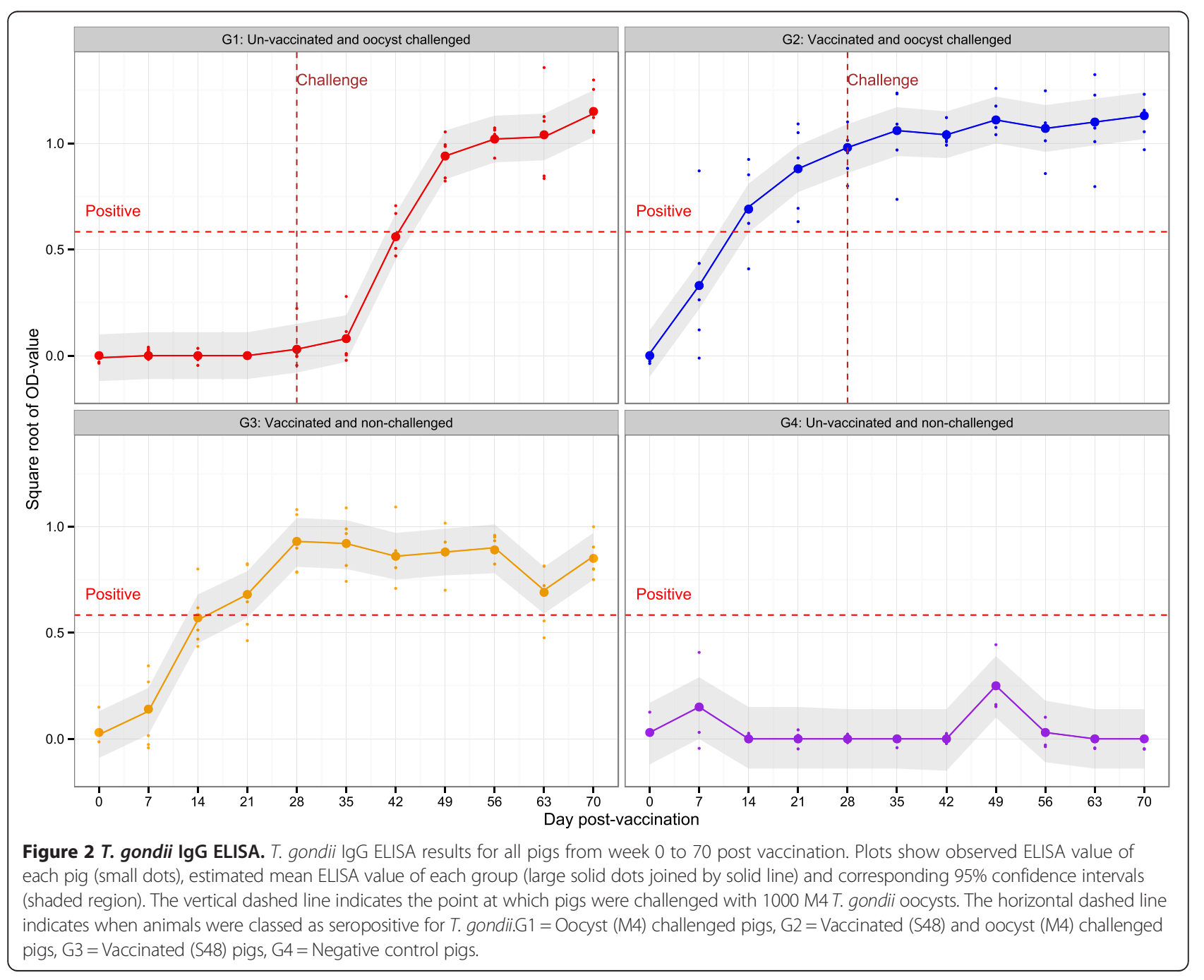


tachyzoites-like structures in the jejunal lymph node from one animal from G1 and in the retromandibular lymph node of another animal from G2.

\section{Mouse bioassay}

During the 6 week mouse bioassay 75.6\% (68/90) of mice survived until the end of the experiment. Twenty two of 90 mice $(24.4 \%)$ had to be euthanised due to the manifestations of clinical signs of $T$. gondii infection (ruffled coat, reluctance to move). All of these mice had received tissues from G1 pigs (unvaccinated and challenged with M4 T. gondii oocysts). From G1 mice, four animals were euthanised on day 11 of the experiment, and a further 18 mice were euthanised on day 12 , which left a total of $51.1 \%(23 / 45)$ of mice surviving until the end of the experiment (day 42) (see Figure 3A). In contrast, all mice, which received tissues from G2 pigs (vaccinated with S48 and challenged with M4 T. gondii oocysts), survived to the end of the experiment, resulting in $100 \%(45 / 45)$ survival of mice (Figure 3A). It was therefore not surprising that the independent twosample log-rank test of the censored survival data until day 42 of the experiment showed strong evidence $(p<$ 0.001 ), that mice which received porcine tissues from G2 pigs had a higher probability of survival across the entire range of the experimental period compared with mice which received G1 porcine tissues.

For mice that received tissues from G1 pigs, there was evidence that the mean proportion of mice that survived, differed between the porcine tissues (brain, food and other) that they were inoculated with (Figure 3B). Among them, 40\% (6/15), 26.7\% (4/15) and 86.7\% (13/ 15) of mice survived when they were inoculated with "brain", "other" and "food" tissues, respectively. Mice that received "food" tissues had a statistically significantly higher chance of surviving during the entire study period compared with mice that received "brain" $(p=$ $0.018)$ and "other" $(p<0.001)$ tissues. The probability of survival between the mice that received "brain" and "other" tissues did not differ significantly $(p=0.340)$.

\section{Detection of T. gondii DNA from mouse brains following bioassay}

Brains from the 22 mice which were euthanised prior to the end of the six week mouse bioassay (all fed with G1 pig tissues - as shown in Figure 4), were positive by ITS1 n-PCR and T. gondii DNA was detected within the brains of all 22 mice. When DNA was extracted from the brains of the remaining 66 mice (which did not show any signs of $T$. gondii infection and were euthanised on day 42 of the bioassay), an additional two mice, which had been inoculated with brain tissue from G1 pigs, were identified as ITS1 positive. Therefore, $T$. gondii DNA was detected in $53.3 \%(24 / 45)$ of mouse brains
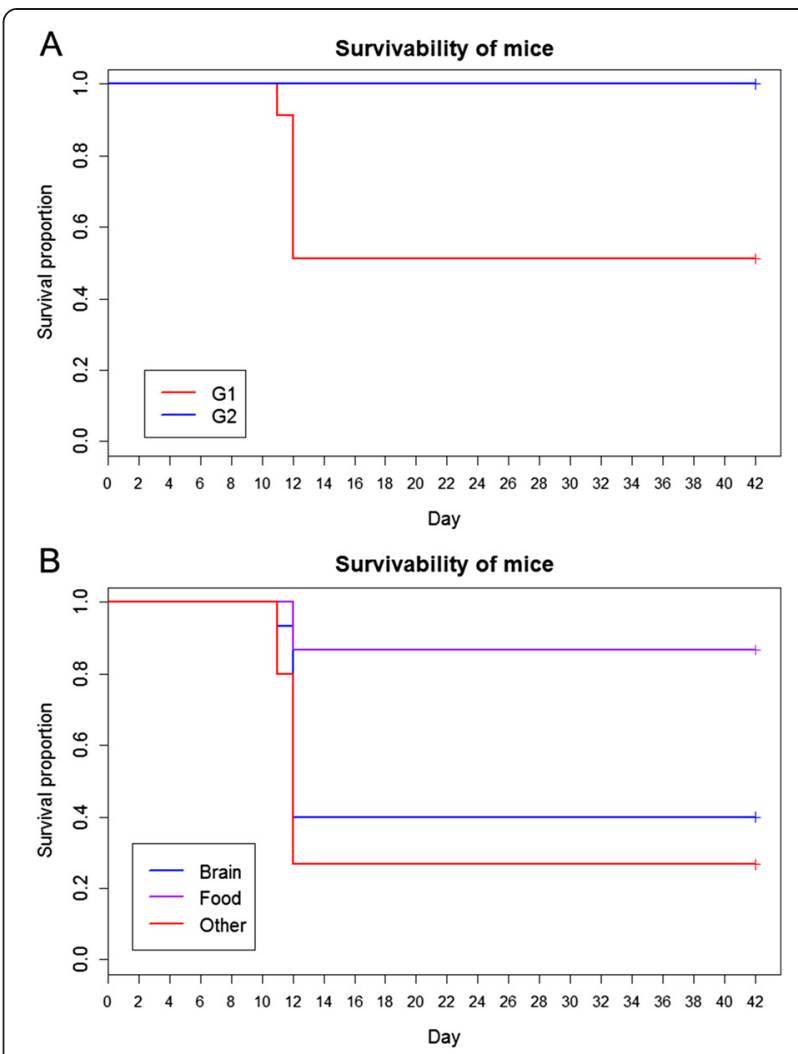

Figure 3 Kaplan-Meir survival curves. Kaplan-Meir survival curve in relation to vaccination highlighting proportion of mice that survived when fed tissues from G1 and G2 pigs (A). All mice which received tissues from vaccinated and challenged pigs (G2) survived (blue line) until the end of the bioassay (day 42). Only $51.1 \%$ of mice which received tissues from unvaccinated and challenged pigs (G1) survived until the end of the bioassay (day 42). G1 = Mice inoculated with porcine tissues from pigs which were vaccinated (\$48) and oocyst (M4) challenged. G2 = Mice which were inoculated with porcine tissues from pigs which were oocyst (M4) challenge. KaplanMeir survival curve from mice fed different tissues from pigs in G1 highlighting proportion of mice that survived when fed different tissue types (brain, food and other) (B). Brain = brain tissue, Food= pooled sample of chop, loin, left tricep and left semitendinosus, Other = pooled sample of diaphragm, heart, tongue and masseter.

which had been inoculated with tissues from G1 pigs (see Table 2). In contrast, 100\% (45/45) of mice, which had been inoculated with tissues from G2 pigs, tested negative for $T$. gondii by ITS1 PCR (see Table 2).

\section{Detection of $T$. gondii DNA from bioassay inocula}

Homogenised and acid/pepsin digested pig tissues used as inocula to challenge the mice were tested for the presence of $T$. gondii DNA. All inocula $(n=30)$ were tested ("food", "brain" and "other" per pig, giving 15 inocula per experimental group), using both the $T$. gondii ITS1 PCR and the T. gondii 529 bp qPCR. T. gondii DNA could not be detected in any of the inocula $(0 / 15)$ generated from G2 pigs by either of conventional $T$. 


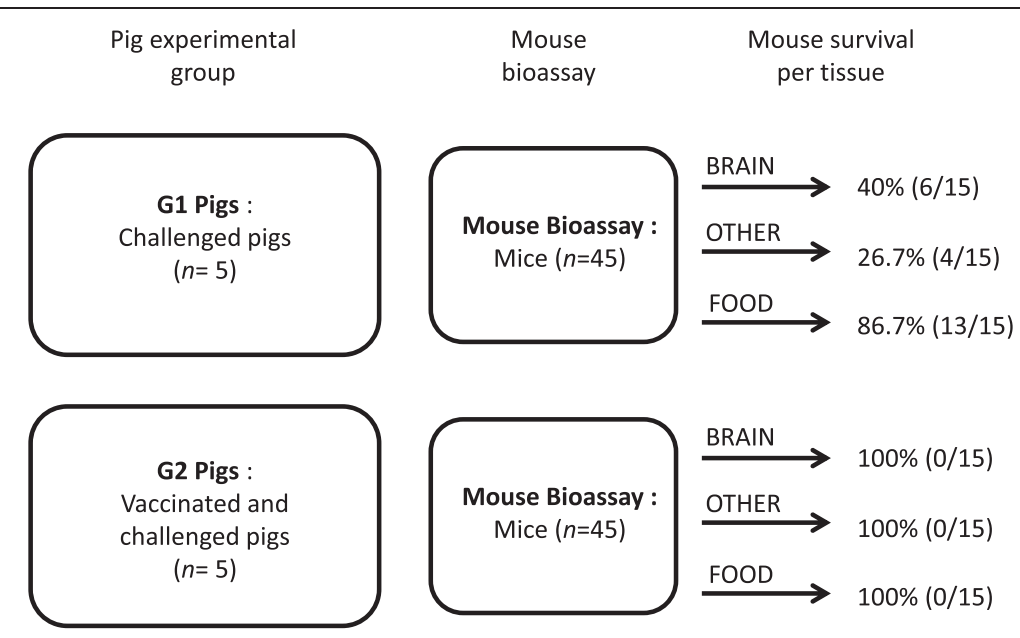

Figure 4 Mouse survival rate related to specific porcine tissues (experimental groups G1 and G2). All mice which received homogenised tissue from G2 pigs survived. Not all mice which received porcine tissues from G1 pigs survived, with only $40 \%$ of mice which received brain tissue surviving, followed by $26.7 \%$ which received food and $86.7 \%$ receiving other.

gondii ITS1 n-PCR, or 529 bp qPCR (Table 3). The results from the ITS1 n-PCR showed that 7/15 (46.7\%) of inocula comprised of tissues from pigs in G1 were positive for T. gondii DNA (Table 4). Of the seven positives, four were from the inocula containing "brain" tissue, two from the inocula comprised of the "other" tissues and one from the inocula comprised of the "food" tissues (Table 4). However, among inocula generated with tissues from G1 pigs, detection of T. gondii DNA using the qPCR was slightly lower compared with detection using the ITS1 n-PCR, with 6/15 (40.0\%) of inocula testing positive by qPCR (compared to $7 / 15$ (46.7\%) by ITS1 PCR) (see Table 4). Inocula, which were used for bioassay in mice, with subsequent detection of $T$. gondii DNA directly from mouse brain, detects the greatest number of $T$. gondii positive tissue samples, resulting in 9/15 (60\%) of pig tissue inocula testing positive. Four of these positives were detected in inocula comprised of "brain", four from "other" and one from "food" tissues. Considering the data on bioassay results, there was however no evidence that mean proportions of detectable infected and non-infected samples differed between tissues (global $p=0.201$ ).

\section{Comparison of molecular detection methods for} detection of $T$. gondii DNA using bioassay inocula

Two molecular tests (T. gondii 529 bp qPCR and the $T$. gondii specific n-ITS1 PCR) were compared for their detection of parasite DNA from bioassay inocula using a gold standard test. The detection of T. gondii DNA directly from mouse brains following bioassay was considered as the "gold standard" as parasite DNA was detected in 9 out of 15 samples (60\%) (see Table 4). The qPCR detected 6 out of 15 samples (40\%), compared with 7 out of 15 samples (46.7\%) by ITS1-PCR. Results showed that both qPCR $(p=0.250)$ and ITS1-PCR $(p=$ $0.500)$ are in agreement with the gold standard test. However, the estimate of sensitivity of the ITS1 PCR (0.78 with $95 \%$ confidence interval: $0.40,0.97)$ was higher compared with the 529pb qPCR (0.67 with 95\% confidence interval: $0.30,0.93)$. The estimates of specificity and 95\% confidence interval for both tests were identical for both methods $(1.00 ; 0.77,1.00)$.

\section{Mouse serology and pathology}

Serum samples from all mice $(n=90)$ were collected at post mortem and tested for the presence T. gondii IgG

Table 2 Summary of $T$. gondii DNA detected by ITS1 PCR in mouse brains following bioassay with porcine tissues

\begin{tabular}{lllll}
\hline Mouse Group & ITS1 positive mice $(\mathbf{n})$ & \% ITS1 positive & ITS1 negative mice $(\mathbf{n})$ & \% ITS1 negative \\
\hline $\mathbf{1}$ & $24^{\mathrm{a}}$ & 53.3 & 21 & 46.7 \\
$\mathbf{2}$ & 0 & 0 & 45 & 100 \\
TOTAL & 24 & 26.7 & 66 & 73.3 \\
\hline
\end{tabular}

${ }^{a}=$ including two mice which survived until the end of the end of the experiment (42 dpi).

$1=$ mice inoculated with porcine tissues from oocyst challenged pigs.

$2=$ mice inoculated with porcine tissues from vaccinated and oocyst challenged pigs. 
Table 3 Detection of $T$. gondii DNA from bioassay inocula (homogenised tissues) compared to detection of $T$. gondii DNA from mouse brain (bioassay) from vaccinated and oocyst challenged pigs (G2)

\begin{tabular}{lllllllllll}
\hline Pig No. & \multicolumn{3}{c}{ Brain } & \multicolumn{3}{c}{ Other } & \multicolumn{3}{c}{ Food } \\
& qPCR & ITS1 & Bio & qPCR & ITS1 & Bio & qPCR & ITS1 & Bio \\
\hline $\mathbf{8 2 0}$ & - & - & - & - & - & - & - & - & - \\
$\mathbf{8 2 1}$ & - & - & - & - & - & - & - & - & - \\
$\mathbf{8 2 2}$ & - & - & - & - & - & - & - & - & - \\
$\mathbf{8 2 3}$ & - & - & - & - & - & - & - & - & - \\
$\mathbf{8 2 4}$ & - & - & - & - & - & - & - & - & - \\
Positives & $0 / 5$ & $0 / 5$ & $0 / 5$ & $0 / 5$ & $0 / 5$ & $0 / 5$ & $0 / 5$ & $0 / 5$ & $0 / 5$ \\
\hline
\end{tabular}

Results from the 529 bp T. gondii qPCR (qPCR), conventional T. gondii ITS1 PCR (ITS1) and ITS1 PCR from mouse brain - bioassay result (Bio), from vaccinated and oocyst challenged pigs (G2).

antibodies using the commercially available ID vet ELISA. The majority of mice $(98.9 \%, 89 / 90)$ tested seronegative (with seropositivity $(\mathrm{SP}) \leq 3 \%$ ), which included all 22 mice euthanised due to signs of T. gondii infection. One mouse (C11-1), which was inoculated with brain tissue from a pig G1 pig, tested positive by ITS1 PCR and survived until the end of the experiment (day 42), was classified as seronegative (23\% SP). However, the percentage seropositivity of this mouse was much higher than that of the remaining 89 seronegative mice. Only one mouse (C11-2), which was also inoculated with brain tissue from a pig in G1, tested positive for T. gondii IgG (189\% SP, this mouse was euthanised at the end of the experiment (day 42) and tested positive by ITS1 PCR.

The pathological results completed on half of each mouse brain $(n=90)$ could not identify any differences between mice inoculated with different porcine tissues (brain, food and other), with the exception of three animals, where tissue cysts were observed (C11-2, C14-3 and $\mathrm{C} 18-2$ ), which had been inoculated with tissues from pigs in G1.

Table 4 Detection of $T$. gondii DNA from bioassay inocula (homogenised tissues) compared to detection of $T$. gondii DNA from mouse brain (bioassay) from oocyst challenged pigs (G1)

\begin{tabular}{llllllllll}
\hline Pig No. & Brain & \multicolumn{3}{c}{ Other } & \multicolumn{3}{c}{ Food } \\
& qPCR & ITS1 & Bio & qPCR & ITS1 & Bio & qPCR & ITS1 & Bio \\
\hline $\mathbf{8 2 5}$ & + & + & + & - & - & - & - & - & - \\
$\mathbf{8 2 6}$ & + & + & + & + & - & + & - & - & - \\
$\mathbf{8 2 7}$ & + & + & + & - & - & + & - & - & - \\
$\mathbf{8 2 8}$ & + & + & + & - & + & + & - & - & - \\
$\mathbf{8 2 9}$ & - & - & - & - & + & + & + & + & + \\
Positives & $4 / 5$ & $4 / 5$ & $4 / 5$ & $1 / 5$ & $2 / 5$ & $4 / 5$ & $1 / 5$ & $1 / 5$ & $1 / 5$
\end{tabular}

Results from the $529 \mathrm{bp} T$. gondii qPCR (qPCR), conventional T. gondii ITS1 PCR (ITS1) and ITS1 PCR from mouse brain - bioassay result (Bio), from oocyst challenged pigs (G1).

\section{Discussion}

The results from this work clearly show that vaccination with a live attenuated strain of the parasite (S48) and subsequent challenge with $T$. gondii oocysts (M4) in pigs is successful in significantly reducing infective tissue cysts from establishing within porcine tissues, as shown by the mouse bioassay. Mouse survival rates from the bioassay at day 42 pi show a clear difference between those mice inoculated with tissues from pigs in G1 (unvaccinated and oocyst challenged pigs), compared with mice inoculated with tissues from pigs in G2 (vaccinated and oocyst challenged pigs) (Figure 3A). In our experience, infection with the M4 strain (type II) of $T$. gondii is likely to cause clinical signs in Porton mice, where viable tissue cysts present in the inocula (homogenised porcine tissue), generally results in severe clinical signs in mice and euthanasia is required. Therefore, in this study, reporting of mouse survival was important as it was a good indicator of the presence or absence of viable tissue cysts. It has previously been reported that as little as one tissue cyst (containing possibly thousands of infective bradyzoites) is enough to cause infection [39]. Other research, which has studied the vaccination of pigs to reduce tissue cyst burden, has mainly focused on microscopic identification of tissue cysts and/or detection of parasite DNA from mice used in the bioassay [21,23-25,35,40,41]. In these studies there is no detailed information about mouse survival and clinical signs of T. gondii during the bioassay itself. In fact, very few $T$. gondii studies, which incorporate mouse bioassays to assess the viability of tissue cysts, describe mouse survival rates in any detail. However, this might also be related to the strain of mouse used, as Swiss Webster mice (although susceptible to $T$. gondii) are generally thought to be more resistant to the parasite and are less likely to show clinical signs of infection. Research by Pena et al. [42], have reported mouse mortality rates following bioassay of cat tissues in relation to the genotype of $T$. gondii present. Our current research describes mouse survival rates using a Kaplan-Meir survival curve. Mouse survival provides an indication of the viability of $T$. gondii tissue cysts, however, this data is lacking in the majority of studies which incorporate mouse bioassay.

In addition to mouse survival rates, $T$. gondii DNA was not detected in any mice $(0 / 45)$ following challenge with inocula comprised of porcine tissues from G2 pigs (vaccinated and oocysts challenged pigs) (Table 3), whilst parasite DNA was detected in 53.3\% (24/45) of mice following challenge with inocula from G1 pigs (non-vaccinated oocyst challenged) (Table 4). Other research, investigating vaccination and $T$. gondii challenge of pigs and subsequent mouse bioassay, have focused only on the microscopic detection of tissues cysts from mouse brain following bioassay $[18,21,25,41]$, rather than detection of 
T. gondii DNA from the mouse brain. Therefore, as the pathology results from the current research provide limited information, it is difficult to draw direct comparisons between these vaccination and challenge experiments and the current research. However, as $100 \%$ of mice from the bioassay survived following inoculation with tissues from pigs vaccinated and oocyst challenge, vaccination with S48 tachyzoites appears to be a very promising approach for reducing parasite burden in porcine tissues. Previous research into vaccination of pigs against $T$. gondii, which have incorporated mouse bioassay, have not shown such a protective response against tissue cyst formation in mouse brains. For example, following immunisation of pigs with crude $T$. gondii rhoptry proteins with Quil-A as an adjuvant, da Cunha et al. [41] found only partial protection from formation of tissue cysts in mouse brains following bioassay with porcine tissues, with the parasite detected in $5 / 11$ (45.4\%) mice in the vaccinated and challenged group. In a similar experiment, partial protection was observed by Garcia et al. [21], who used T. gondii rhoptry proteins and immunostimulating complexes (ISCOMS) as an adjuvant to vaccinate pigs. Dubey et al. [25] tested a vaccine incorporating a low dose of irradiated $T$. gondii oocysts, and although fewer tissue cysts were observed in mice, cysts were detected in 45/110 (40.9\%) of mice which had received porcine tissues from vaccinated pigs. In other work by Dubey et al. [18] using tachyzoites of a nonpersistent strain of $T$. gondii $(\mathrm{RH})$ to vaccinate pigs, only partial protection was described, with fewer tissues cysts in mice inoculated with porcine tissues. Similar results were also reported using the T. gondii TS-4 mutant to vaccinate pigs $[43,44]$, where the vaccine alone did not persist, however only partial protection was observed following challenge with GT-1 oocysts. In summary, apart for the research described within this manuscript, none of the literature currently available can describe $100 \%$ protection against tissue cyst formation in mice, following bioassay of porcine tissues.

From the current results, it appears that vaccination with $\mathrm{S} 48$ alone does not induce tissue cyst formation in porcine tissues, as results from the mouse bioassay, which included inocula with porcine tissues from the vaccinated and challenged animals, were all PCR negative for $T$. gondii. If a positive had been obtained from this group, the experiment had been designed to verify whether infection was due to vaccination (S48) or oocyst challenge (M4), as previously shown in lambs [11] (both S48 and M4 are different T. gondii genotypes; S48 = type I, M4 = type II). However, a bioassay using porcine tissues from animals that were vaccinated alone (which was not included in this study), could further support this result.

The results also show that there were differences in survival rates within groups of mice which were inoculated using tissues from G1 pigs, depending on which type of tissue they had been inoculated with (Figure 4). A greater proportion of mice survived which had received porcine inocula from the "food" tissue group (chop, loin, left triceps and left semitendinosus), compared with mice which had received tissues from the "brain" and "other" tissue groups (diaphragm, heart, tongue and masseter). This suggests that there are a greater number of viable parasites within tissues from the "brain" and "other" groups in comparison to tissues that are used routinely for human consumption ("food"). To further confirm this result, $T$. gondii DNA was detected in the brains of all mice which were euthanised due to clinical signs of $T$. gondii infection, with parasite DNA being detected in fewer bioassay mice which had received porcine tissues from the "food" group using tissues from G1 pigs (oocysts challenged pigs), compared with mice which received brain or tissues from the "other" group (Table 4).

In addition, two mice (C11-1 and C11-2), which had been inoculated with tissues from G1 pigs that survived until the end of the experiment (day 42) but also tested positive for T. gondii by detection of parasite DNA in their brains. Accordingly, they were the only two mice which gave a seropositivity of greater than $3 \%$ with the T. gondii IgG ELISA. It is likely that mice, which were euthanised within the first 14 days of the bioassay, which also tested positive for the presence of $T$. gondii DNA in their brains, may only just have started to mount a humoral immune response against the parasite, however, the levels of IgG present were possibly too low to be detected by the ELISA.

Although the T. gondii specific ITS1 PCR was carried out using DNA from individual porcine tissues (brain, chop, loin, left tricep, left semitendinosus, diaphragm, heart, tongue and masseter), parasite DNA could not be detected from any tissues. When comparing this result with a similar study carried out in lambs [11], where $T$. gondii DNA was detected from individual tissues by ITS1 PCR, it is likely that the challenge dose of 1000 oocysts may be too low to be detected by this method. Although 1000 M4 oocysts is more likely to reflect that of a natural infection, it appears that a higher challenge dose (500 000 M4 oocysts as used by [11]), may result in a greater chance of detecting parasite DNA directly from the hosts tissue, without the need for mouse bioassay or detection of parasite DNA from bioassay inocula. In addition, direct detection of parasite DNA from porcine tissues used only $1 \mathrm{~g}$ of starting material, compared with $50 \mathrm{~g}$ of tissue which was prepared for mouse bioassay, therefore, due to the inhomogeneous distribution of parasite tissue cysts there was less chance of detecting the parasite from a smaller $1 \mathrm{~g}$ sample. In the current study, detection of parasite DNA from bioassay inocula was tested by two different molecular techniques; the 
529 bp T. gondii qPCR and the T. gondii ITS1 PCR, to determine which test was more sensitive and whether either technique was as sensitive as mouse bioassay (the "gold standard" assay for detection of viable tissue cysts). Although detection of parasite DNA from mouse brains, following bioassay by ITS1 PCR, detected the greatest number of positive samples there was no evidence of a difference between two molecular tests. However, the sensitivity of the ITS1 PCR was slightly higher than the 529 bp qPCR ( 0.78 and 0.67 respectively). It should also be noted that although these two molecular techniques successfully detect parasite DNA, it is still currently only the mouse bioassay which has the ability to detect viable tissue cysts, and their potential to infect another host. However, perhaps future methodology could employ both molecular detection of the parasite from bioassay inocula in conjunction with mouse bioassay, with a view to reducing the number of mice used within the bioassay, such as magnetic capture qPCR (MC-qPCR) of T. gondii DNA as described by [10]. Histological examination of porcine samples was of limited use to detect the parasite in the porcine tissue or to show differences between the groups of infected mice. However, it showed how vaccination protected against the occurrence of lesions in the brain (perivascular foci) after infection. Immmunohistochemical labelling was also ineffective when trying to localize the parasite, as only in two animals tachyzoites-like structures were detected. These results from pathological studies suggest that these techniques are not adequate when studying parasite distribution in studies where no (porcine experiment) or very similar (murine bioassay) lesions are originated.

Overall, in terms of vaccination to reduce viable tissue cysts in meat, these results are promising, as this is the first description of $100 \%$ protection against tissue cyst formation in mice following mouse bioassay of tissues from pigs. The results also provide an answer to the question raised by the Food Standards Agency, in a recent report published by the Advisory Committee on the Microbiological Safety of Food (ACMSF), the document states that one of the knowledge gaps relevant to a risk assessment in the UK is: "vaccines based on live attenuated strains of tachyzoites are effective in reducing morbidity in sheep but it is not known whether vaccination has any effect on the formation of tissue cysts" [26]. From the current research it is now clear that S48 does have a significant effect in reducing the formation of tissue cysts in pigs. Vaccination of other "high risk" livestock species should be addressed and it has recently been demonstrated that vaccination of sheep with S48 also results in a reduction in the number of ovine tissue samples in which parasite DNA can be detected [11].

\section{Competing interests}

The authors declare that they have no competing interests.

\section{Authors' contributions}

$F K, E A I, A B, J L G$ conceived and designed the experiment; $A B$ and FK conducted the experiment; AB, FK, GC, PMB, JB, FC, JL and JT completed pig post mortem examinations, mouse bioassays, serology and molecular analysis; FK, $A B$ and $M N$ carried out statistical analysis and contributed to the interpretation of data; $A B, F K, M N$ wrote the paper and provided important intellectual content; all authors read and approved the final version.

\section{Acknowledgements}

This project was partially funded by a transnational access project funded through the European Union Seventh Framework Network of Animal Disease Infectiology Research Facilities (NADIR; reference number FP7-228394). AB was funded by the Moredun Foundation. FK, PMB, MN, JT, FC, EAI were supported by the Scottish Government, Rural and Environmental Sciences and Analytical Services (RESAS). JB was supported by CSIC and financed in part by European Social Fund (ESF). JLG was financed by Coordenação de Aperfeiçoamento de Pessoal de Nível Superior (CAPES, BEX 10259-/12-0), Brazil and GC by the Instituto Nacional de Tecnologia Agropecuaria (INATA), Argentina.

\section{Author details}

${ }^{1}$ Moredun Research Institute, Pentlands Science Park, Bush Loan, Midlothian EH26 OPZ, Scotland, UK. ${ }^{2}$ Instituto de Ganadería de Montaña (CSIC-ULE), León, Spain. ${ }^{3}$ Instituto Nacional de Tecnología Agropecuaria (INATA), EEA Balcarce, Argentina. ${ }^{4}$ Departamento de Medicina Veterinária Preventiva, Universidade Estadual de Londrina, Londrina, Brazil. ${ }^{5}$ Biomathematics \& Statistics Scotland, The King's Buildings, Edinburgh EH9 3JZ, Scotland, UK.

Received: 26 January 2015 Accepted: 26 March 2015

Published online: 01 May 2015

\section{References}

1. Kijlstra A, Meerburg B, Cornelissen J, De Craeye S, Vereijken P, Jongert E (2008) The role of rodents and shrews in the transmission of Toxoplasma gondii to pigs. Vet Parasitol 156:183-190

2. Esteban-Redondo I, Maley SW, Thomson K, Nicoll S, Wright S, Buxton D, Innes EA (1999) Detection of T. gondii in tissues of sheep and cattle following oral infection. Vet Parasitol 86:155-171

3. Garcia JL, Innes EA, Katzer F (2014) Current progress toward vaccines against Toxoplasma gondii. Vaccine (Auckl) 4:23-37

4. Tenter AM, Heckeroth AR, Weiss LM (2000) Toxoplasma gondii: from animals to humans. Int J Parasitol 30:1217-1258

5. Swierzy IJ, Muhammad M, Kroll J, Abelmann A, Tenter AM, Luder CG (2014) Toxoplasma gondii within skeletal muscle cells: a critical interplay for foodborne parasite transmission. Int J Parasitol 44:91-98

6. Dubey JP (1988) Long-term persistence of Toxoplasma gondii in tissues of pigs inoculated with T gondii oocysts and effect of freezing on viability of tissue cysts in pork. Am J Vet Res 49:910-913

7. Kijlstra A, Jongert E (2009) Toxoplasma-safe meat: close to reality? Trends Parasitol 25:18-22

8. Dubey JP, Murrell KD, Fayer R, Schad GA (1986) Distribution of Toxoplasma gondii tissue cysts in commercial cuts of pork. J Am Vet Med Assoc 188:1035-1037

9. Aspinall TV, Marlee D, Hyde JE, Sims PFG (2002) Prevalence of Toxoplasma gondii in commercial meat products as monitored by polymerase chain reaction - food for thought? Int J Parasitol 32:1193-1199

10. Opsteegh $M$, Langelaar $M$, Sprong $H$, den Hartog L, De Craeye S, Bokken G, Ajzenberg D, Kijlstra A, van der Giessen J (2010) Direct detection and genotyping of Toxoplasma gondii in meat samples using magnetic capture and PCR. Int J Food Microbiol 139:193-201

11. Katzer F, Canton G, Burrells A, Palarea-Albaladejo J, Horton B, Bartley PM, Pang Y, Chianini F, Innes EA, Benavides J (2014) Immunization of lambs with the S48 strain of Toxoplasma gondii reduces tissue cyst burden following oral challenge with a complete strain of the parasite. Vet Parasitol 205:46-56

12. Cook AJ, Gilbert RE, Buffolano W, Zufferey J, Petersen E, Jenum PA, Foulon W, Semprini AE, Dunn DT (2000) Sources of toxoplasma infection in pregnant women: European multicentre case-control study. European Research Network on Congenital Toxoplasmosis. BMJ 321:142-147 
13. Dubey JP (2008) The history of Toxoplasma gondii-the first 100 years. J Eukaryot Microbiol 55:467-475

14. Kijlstra A, Eissen OA, Cornelissen J, Munniksma K, Eijck I, Kortbeek T (2004) Toxoplasma gondii infection in animal-friendly pig production systems. Invest Ophthalmol Vis Sci 45:3165-3169

15. van der Giessen J, Fonville M, Bouwknegt M, Langelaar M, Vollema A (2007) Seroprevalence of Trichinella spiralis and Toxoplasma gondii in pigs from different housing systems in The Netherlands. Vet Parasitol 148:371-374

16. Lehmann T, Graham DH, Dahl E, Sreekumar C, Launer F, Corn JL, Gamble HR, Dubey JP (2003) Transmission dynamics of Toxoplasma gondii on a pig farm. Infect Genet Evol 3:135-141

17. Hill DE, Haley C, Wagner B, Gamble HR, Dubey JP (2010) Seroprevalence of and risk factors for Toxoplasma gondii in the US swine herd using sera collected during the National Animal Health Monitoring Survey (Swine 2006). Zoonoses Public Health 57:53-59

18. Dubey JP, Urban JF, Jr, Davis SW (1991) Protective immunity to toxoplasmosis in pigs vaccinated with a nonpersistent strain of Toxoplasma gondii. Am J Vet Res 52:1316-1319

19. Djurković-Djaković O, Bobić B, Nikolić A, Klun I, Dupouy-Camet J (2013) Pork as a source of human parasitic infection. Clin Microbiol Infect 19:586-594

20. Innes EA, Bartley PM, Rocchi M, Benavidas-Silvan J, Burrells A, Hotchkiss E, Chianini F, Canton G, Katzer F (2011) Developing vaccines to control protozoan parasites in ruminants: dead or alive? Vet Parasitol 180:155-163

21. Garcia JL, Gennari SM, Navarro IT, Machado RZ, Sinhorini IL, Freire RL, Marana ER, Tsutsui V, Contente AP, Begale LP (2005) Partial protection against tissue cysts formation in pigs vaccinated with crude rhoptry proteins of Toxoplasma gondii. Vet Parasitol 129:209-217

22. Jongert E, Melkebeek V, De Craeye S, Dewit J, Verhelst D, Cox E (2008) An enhanced GRA1-GRA7 cocktail DNA vaccine primes anti-Toxoplasma immune responses in pigs. Vaccine 26:1025-1031

23. Kringel H, Dubey JP, Beshah E, Hecker R, Urban JF, Jr (2004) CpGoligodeoxynucleotides enhance porcine immunity to Toxoplasma gondii. Vet Parasitol 123:55-66

24. Dubey JP, Baker DG, Davis SW, Urban JF, Jr, Shen SK (1994) Persistence of immunity to toxoplasmosis in pigs vaccinated with a nonpersistent strain of Toxoplasma gondii. Am J Vet Res 55:982-987

25. Dubey JP, Lunney JK, Shen SK, Kwok OC (1998) Immunity to toxoplasmosis in pigs fed irradiated Toxoplasma gondii oocysts. J Parasitol 84:749-752

26. AMCSF (2012) Ad Hoc Group on Vulnerable Groups: Risk profile in relation to toxoplasma in the food chain. Advisory Committee on the Microbiological Safety of Food. http://www.food.gov.uk/sites/default/files/ multimedia/pdfs/committee/acmsfrtaxopasm.pdf

27. Buxton D, Innes EA (1995) A commercial vaccine for ovine toxoplasmosis. Parasitology 110:S11-S16

28. Innes EA, Bartley PM, Maley S, Katzer F, Buxton D (2009) Veterinary vaccines against Toxoplasma gondii. Mem Inst Oswaldo Cruz 104:246-251

29. Jacobs L, Remington JS, Melton ML (1960) A survey of meat samples from swine, cattle, and sheep for the presence of encysted Toxoplasma. J Parasitol 46:23-28

30. Dubey JP (1998) Refinement of pepsin digestion method for isolation of Toxoplasma gondii from infected tissues. Vet Parasitol 74:75-77

31. Bartley PM, Wright SE, Zimmer IA, Roy S, Kitchener AC, Meredith A, Innes EA, Katzer F (2013) Detection of Neospora caninum in wild carnivorans in Great Britain. Vet Parasitol 192:279-283

32. Burrells A, Bartley PM, Zimmer IA, Roy S, Kitchener AC, Meredith A, Wright SE, Innes EA, Katzer F (2013) Evidence of the three main clonal Toxoplasma gondii lineages from wild mammalian carnivores in the UK. Parasitology 140:1768-1776

33. Katzer F, Brulisauer F, Collantes-Fernandez E, Bartley PM, Burrells A, Gunn G, Maley SW, Cousens C, Innes EA (2011) Increased Toxoplasma gondii positivity relative to age in 125 Scottish sheep flocks; evidence of frequent acquired infection. Vet Res 42:121

34. Opsteegh M, Swart A, Fonville M, Dekkers L, van der Giessen J (2011) Agerelated Toxoplasma gondii seroprevalence in Dutch wild boar inconsistent with lifelong persistence of antibodies. PLoS One 6:e16240

35. Garcia JL, Gennari SM, Machado RZ, Navarro IT (2006) Toxoplasma gondii: Detection by mouse bioassay, histopathology, and polymerase chain reaction in tissues from experimentally infected pigs. Exp Parasitol 113:267-271

36. Benjamini $Y$, Hochberg $Y$ (1995) Controlling the false discovery rate: a practical and powerful approach to multiple testing. J R Stat Soc Ser B Stat Methodol 7:289-300
37. Harrington DP, Fleming TR (1982) A class of rank test procedures for censored survival data. Biometrika 69:553-566

38. R-Core-Team (2014) A language and environment for statistical computing. Foundation for Statistical Computing, Vienna, Austria. http:/www.R-project.org/

39. Dubey JP, Lindsay DS, Speer CA (1998) Structures of Toxoplasma gondii tachyzoites, bradyzoites, and sporozoites and biology and development of tissue cysts. Clin Microbiol Rev 11:267-299

40. Freire RL, Navarro IT, Bracarense APFRL, Gennari SM (2003) Vaccination of pigs with Toxoplasma gondii antigens incorporated in immunostimulating complexes (iscoms). Arq Bras Med Vet Zootec 55:388-396

41. da Cunha IA, Zulpo DL, Bogado AL, de Barros LD, Taroda A, Igarashi M, Navarro IT, Garcia UL (2012) Humoral and cellular immune responses in pigs immunized intranasally with crude rhoptry proteins of Toxoplasma gondii plus Quil-A. Vet Parasitol 186:216-221

42. Pena HF, Soares RM, Amaku M, Dubey JP, Gennari SM (2006) Toxoplasma gondii infection in cats from Sao Paulo state, Brazil: seroprevalence, oocyst shedding, isolation in mice, and biologic and molecular characterization. Res Vet Sci 81:58-67

43. Lindsay DS, Blagburn BL, Dubey JP (1993) Safety and results of challenge of weaned pigs given a temperature-sensitive mutant of Toxoplasma gondii. J Parasitol 79:71-76

44. Pinckney RD, Lindsay DS, Blagburn BL, Boosinger TR, McLaughlin SA, Dubey JP (1994) Evaluation of the safety and efficacy of vaccination of nursing pigs with living tachyzoites of two strains of Toxoplasma gondii. J Parasitol 80:438-448

\section{Submit your next manuscript to BioMed Central and take full advantage of:}

- Convenient online submission

- Thorough peer review

- No space constraints or color figure charges

- Immediate publication on acceptance

- Inclusion in PubMed, CAS, Scopus and Google Scholar

- Research which is freely available for redistribution 